\section{Original research presented at the ABCD 2014 Autumn meeting}

\author{
The Autumn meeting of $A B C D$ was held at the Royal College of \\ Physicians, London. As ever, some delegates shared their latest \\ original research as poster presentations. To give a flavour of the \\ data presented, we provide a précis of each abstract. We hope \\ you find our brief report interesting, and look forward to hearing \\ about your own research at a future meeting.
}

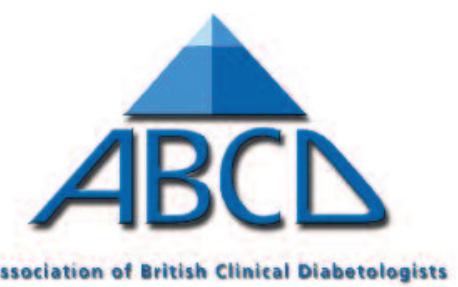

Early 'real world' data on dapagliflozin: effective, but more side-effects than in clinical trials (McGovern AP et al) ${ }^{1}$

This retrospective case-note audit of all 96 outpatients started on dapagliflozin in a specialist clinic of a London hospital showed that $42 \%$ had an $\mathrm{HbA}_{1 \mathrm{c}}$ reduction $\geq 1 \%$, while $29 \%$ had no reduction; $15 \%$ had weight loss $\geq 5 \mathrm{~kg}$ and $3 \%$ had weight loss $\geq 10 \mathrm{~kg}$, while $24 \%$ had no weight reduction. Genital candidiasis, nocturia, and polyuria were the most common sideeffects. The rate of discontinuation of dapagliflozin for side-effects (22\%) was higher the $3-4 \%$ reported in trials (3-4\%). Of people who tolerated dapagliflozin, $38 \%$ could stop or reduce at least one other diabetes medication. Dapagliflozin is effective in real world clinical practice, with additional benefits beyond glycaemic control, but induced more side-effects compared with patients in previous clinical trials. Now published in BJDVD as a full paper: http:// bjdvd.com/index.php/bjdvd/article/view/36.

Measuring urinary C-Peptide:creatinine ratio (UCPCR) contributes importantly to long-term diabetes management (Naqvi AR et $a l)^{2}$

This 2-year retrospective audit showed that measuring UCPCR (within 5 years of diabetes diagnosis in 25 patients and after this time in the remainder) contributed to the management plan for $36 / 38$ patients over 2 years, providing confirmation of the diagnosis in 30 patients. The test is most useful in diabetes of longer-standing, as C-peptide levels decline more rapidly for type 1 diabetes than type 2 diabetes over this time period. Also, elevated C-peptide levels five or more years post-diagnosis help to distinguish between type 1 diabetes and MODY. Knowing the patient's insulin secretory status is important for constructing care plans and may assist in the management of a patient presenting with diabetic ketoacidosis.
Elevated prevalence of clinically significant chronic liver disease among older, community-dwelling type 2 diabetes patients (Morling JR et $\mathrm{al})^{3}$

Data over 6 years from 939 participants in the Edinburgh Type 2 Diabetes Study showed that 36 patients had new or existing chronic liver disease, including 35 (3.7\% overall) with cirrhosis, 9 (1.0\%) with hepatocellular carcinoma and $11(1.2 \%)$ with oesophageal varices. Only $7 / 15$ subjects with incident chronic liver disease during the follow-up were identified by an extensive liver assessment. Abnormal liver enzymes predicted chronic liver disease (IRR 5.7, 95\% CI 2.0 to $16.0, p=0.001)$, but hepatic steatosis did not. Systemic inflammation, steatohepatitis and hepatic fibrosis were also associated with developing incident chronic liver disease. The incidence of chronic liver disease in this type 2 diabetic population was higher than for the general population and we need to identify patients at risk to facilitate timely intervention and follow-up.

Prognostic importance of glucose levels on admission to hospital (McAllister DA et $a l)^{4}$

Hyperglycaemia on admission is common in people without known diabetes, but the subsequent risk of type 2 diabetes is unknown. This study used linked databases (SCI-DC national register of patients with diabetes, a national hospitalisation database and regional biochemistry results databases) to answer this question in 86,634 patients aged $\geq 40 \mathrm{y}$ admitted between 2004 and 2008. The 3-year risk of diabetes (by logistic regression) increased from $<1 \%$ for venous glucose $<5$ $\mathrm{mmol} / \mathrm{L}$ to $2.6 \%$ at $7 \mathrm{mmol} / \mathrm{L}, 9.9 \%$ at 11.1 $\mathrm{mmol} / \mathrm{L}$ and about $15 \%$ at $15 \mathrm{mmol} / \mathrm{L}$. Information about admission glucose levels can quantify the risk of subsequent diabetes and contribute to their subsequent management. This study has now been published (McAllister DA et al, Plos Med 2014;11:e1001708).
The proteomic classifier CKD273 is distinguishes between different severities of renal dysfunction in type 2 diabetes albuminuria (Currie G et al) ${ }^{5}$

This study involved 45 type 2 diabetes patients (15 each with normoalbuminuria, microalbuminuria and diabetic nephropathy). The groups were similar for age and BP and were similarly at high cardiovascular risk, based on Framingham scores, ASSIGN scores, Alx@75 and carotid intima-media thickness (the last two were independent of eGFR). Nevertheless, mean CKD273 differed $(p=0.002)$ between normoalbuminuria $(-0.17 \pm 0.37)$, microalbuminuria $(0.42$ $\pm 0.37)$ and diabetic nephropathy $(0.77 \pm$ $0.43)$, without significant correlation with measures of vascular damage. Thus, CKD273 distinguished between categories of diabetic renal dysfunction independently of vascular phenotype.

A case of devastating myopathy in a statin-treated type 2 diabetes patient (Whincup $\mathrm{C}$ et $\mathrm{al})^{6}$

A type 2 diabetes patient presented to Accident and Emergency with a 3-month history of worsening myalgia, generalised weakness, fatigue, fever, night sweats, dysphagia and $4 \mathrm{~kg}$ weight loss, previously treated as a viral illness but starting soon after commencing simvastatin. He had markedly reduced power in the proximal muscles bilaterally with normal power in peripheral muscle groups; sensation, reflexes and tone were normal. Creatinine kinase was grossly elevated (24,514 iu). Electromyography, MRI and biopsy revealed myopathic changes with an acute inflammatory response in the proximal muscles, oedema and severe acute myopathy with necrosis and myophagocytosis. He was positive for anti-HMG-Co-A antibody. A full recovery followed tretatment with i.v. methyprednisolone and later high dose prednisolone followed by i.v. immunoglob- 
ulin. Statin cessation may not result in symptom relief if the patient has developed autoantibodies to HMG-Co-A reductase. Patients and clinicians should be aware of the side effects of statins and weigh their risks and benefits.

How to improve your insulin prescription chart (Dashora $U$ et al) ${ }^{7}$

Insulin related prescription errors are common. The Joint British Diabetes Societies for Inpatient care (JBDS) organised a national competition for the 'best in class' insulin prescription chart (41 trusts entered). Strengths of the best charts included a 'very practical three page fold-out', 'uncluttered, 'easy to understand', 'inclusion of sc insulin in main drug chart', 'separate charts for the various IV regimens', 'inclusion of pre admission regimen', 'storage advice on chart', 'advice on moving insulin WITH patients and self-administration', 'advise on non return valves for iv insulin infusion' and 'instruction on making up IV infusion.'. Suggestions for improving charts included 'prescribing by brand name', 'integrated blood glucose monitoring', 'chart for hyperkalaemia', 'colour coding for different charts', 'better guidance on when to start which regimen', 'better reference to units (rather than $U$ ) and 'adequate space for monitoring and dose changes.' Look out for an upcoming article on this subject in BJDVD.

Mixed early impressions of degludec (Crane J et al) ${ }^{8}$

Twenty-four patients received degludec over 15 months (18 with type 1 diabetes, 3 with type 2 diabetes and 3 with pancreatic failure; age 19-75 y and diabetes duration 1-48 y). The intention was to address issues of adherence and hyperglycaemia $(n=17)$, recurrent hypos ( $n=6$; hypo frequency reduced in two patients and increased in one patient) or to improve flexibility of injection timing $(n=1)$. Mean $\mathrm{HbA}_{1}$ c was unchanged between initiation and 10 months (11.1\%/98 $\mathrm{mmol} / \mathrm{mol}$ at each time). For 5 patients with recurrent DKA, the frequency decreased for one and increased for another, with insufficient follow-up for the remaining three. There was one admission for severe, prolonged hypoglycaemia (suggesting a need for caution with ultra-long acting insulin in patients at increased hypo risk). Overall, there was no change in extremes of glycaemia.

Using degludec instead of U500 in a patient with severe insulin resistance due to antibodies (Acharya et al) ${ }^{9}$

This was a case report of an Asian male, age 56 y, with diabetes for 30 y. In 2011, his U100 insulin requirement increased to 3.8 $\mathrm{U} / \mathrm{kg}$ (300 U/day) and insulin antibodies were positive. He was changed to Insulin U500, titrated to 35/20/30 U/day (insulin $425 \mathrm{U} /$ day). In 2013, he was changed to degludec U200 (total 62 U QD). HbA 1c fell from $11 \%$ to $9 \%$, antibody levels normalised and weight remained stable. Degludec cost $\mathrm{f} 120 /$ month vs. $\mathrm{f} 200 /$ month for U500. Our case report suggests that degludec U100/200 could be considered in patients with severe insulin resistance.

Consider primary aldosteronism as a possible cause of refractory hypertension (Hussain S et al) ${ }^{10}$

A 61 year old, previously healthy Caucasian man presented with abdominal pain, general tiredness and refractory hypertension, despite an ACE inhibitor, calcium channel blocker and diuretics. Local gastroenterologists reported a normal oesophagogastroduodenoscopy and colonoscopy, but a CT scan and further investigation found a $1.5 \mathrm{~cm}$ right-sided adrenal adenoma and a raised plasma aldosterone/renin ratio, consistent with primary hyperaldosteronism (Conn's Syndrome). Adrenal vein sampling revealed raised contralateral aldosterone levels, ruling out surgery. Spironolactone alone has maintained normotensive blood pressure, and the patient is delighted to be free of polypharmacy despite a small degree of drug-related gynaecomastia. This case demonstrates the need to further investigate the causes of hypertension, in particular unremitting hypertension despite antihypertensive polypharmacy.

\section{Abstract titles and authors}

1. McGovern AP, Dutta N, Watters K, Munro N, FeherM. Early 'real world' data on da- pagliflozin: effective glucose control, blood pressure reduction, weight loss and reduced medication burden. From the Beta Cell Centre for Diabetes, Chelsea and Westminster Hospital, the Diabetes Therapies Evaluation Network, the Department of Health Care Management and Policy, University of Surrey and the .Clinical Sciences Research Institute, Warwick University

2. Naqvi AR, Winocour P, Lungley A, and Batchelor B. Role of Urine C-Peptide Creatinine ratio in the management of Diabetes. From the East \& North Hertfordshire NHS Trust.

3. Morling JR, Fallowfield JA, Guha IN, Williamson RM, Ali M, Nee LD. The burden and development of clinically significant chronic liver disease in older people with type 2 diabetes: The Edinburgh Type 2 Diabetes Study. From the University of Edinburgh.

4. McAllister DA, Hughes KA, Lone N, Mills NL, Sattar N, McKnight J, Wild SH. Stress hyperglycaemia in hospitalised patients and their 3-year risk of diabetes: A Scottish retrospective cohort study, From the Centre for Population Health Sciences, University of Edinburgh.

5. Currie G, Friar M, Drummond R, Mullen W, Mischak $H$, Delles C. Urinary proteomics for diagnosis of nephropathy and subclinical vascular damage in type 2 diabetes. From the University of Glasgow.

6. Wincup C, Chopra A, Nikookam Y, Hussain S, Debrera G, Smithers E, Casey E. Diabetes mellitus, statins and devastating myopathy. From the deptartments of Rheumatology, Diabetes and Endocrinology and Anaesthesia, King George Hospital. Barking, Havering and Redbridge University Hospitals NHS Trust.

7. Dashora U, Castro E, Stanisstreet D. Best in class insulin prescription charts. From Conquest Hospital, St Leonards on Sea.

8. Crane J, Pender S. Experience of Tresiba at Guy's and St Thomas' Hospital. From Guy's \& St Thomas' NHS Trust, London.

9. Acharya J, Munro N, Watters K, Scarle S, Feher MD. Insulin Degludec, an alternative to Insulin U500, in severe insulin resistance due to antibodies. From the Beta Cell Diabetes Centre, Chelsea and Westminster Hospital, London.

10. Hussain S, Chopra A, Abdin A, Grainger S, Matson M, Casey E, Nikookam K. A final resolution following years of polypharmacy From St Bartholomew's Hospital, London and King George Hospital. Barking.

http://dx.doi.org/10.15277/bjdvd.2015.049 Br J Diabetes Vasc Dis 2015:15:99-100 